\title{
LOCAL BEHAVIOR NEAR QUASI-PERIODIC SOLUTIONS OF CONFORMALLY SYMPLECTIC SYSTEMS
}

\author{
RENATO C. CALLEJA, ALESSANDRA CELLETTI, AND RAFAEL DE LA LLAVE
}

ABSTRACT. We study the behavior of conformally symplectic systems near rotational Lagrangian tori. We recall that conformally symplectic systems appear for example in mechanical models including a friction proportional to the velocity.

We show that in a neighborhood of these quasi-periodic solutions (either transitive tori of maximal dimension or periodic solutions), one can always find a smooth symplectic change of variables in which the time evolution becomes just a rotation in some direction and a linear contraction in others. In particular quasi-periodic solutions of contractive (expansive) diffeomorphisms are always local attractors (repellors). We present results when the systems are analytic, $C^{r}$ or $C^{\infty}$. We emphasize that the results presented here are non-perturbative and apply to systems that are far from integrable; moreover, we do not require any assumption on the frequency and in particular we do not assume any non-resonance condition.

We also show that the system of coordinates can be computed rather explicitly and we provide iterative algorithms, which allow to generalize the notion of "isochrones". We conclude by showing that the above results apply to quasi-periodic conformally symplectic flows.

\section{Contents}

1. Introduction 2

2. Definitions and statement of the results 3

2.1. Conformally symplectic systems 3

2.2. Invariant tori $\quad 5$

2.3. Local models near an invariant torus 6

Department of Mathematics and Statistics, McGill University, 805 Sherbrooke Street West Montreal, Quebec, Canada, H3A 2K6

Department of Mathematics, University of Roma Tor Vergata, Via della Ricerca Scientifica 1, 00133 Roma (Italy)

School of Mathematics, Georgia Institute of Technology, 686 Cherry St., Atlanta GA. 30332-1160

E-mail addresses: calleja@math.mcgill.ca, celletti@mat.uniroma2.it, rafael.delallave@math.gatech.edu.

2000 Mathematics Subject Classification. 70K43, 70K20, 34D35.

Key words and phrases. Dissipative systems, conformal mappings, quasi-periodic solutions, attractors.

R.L. was partially supported by NSF grant DMS-091389. R.C. was partially supported by NSERC, the CRM (Montréal), and by the NQRNT-PBEEE grant. 
3. Proof of Theorem 2

3.1. A system of coordinates and an approximate solution of (2.13) in a $\begin{array}{lr}\text { neighborhood of the torus } & 9\end{array}$

3.2. Making the approximate conjugation symplectic 11

3.3. End of the proof of Theorem $2 \quad 13$

4. Some complements and extensions of the proof 14

4.1. Finite differentiable and $C^{\infty}$ results 14

4.2. Relation with the theory of isochrones 16

4.3. Convergence of the series by the majorant method 19

5. Extension of the results to quasi-periodic perturbations 22

5.1. Main construction 23

5.2. Some elementary consequences of the construction 23

$\begin{array}{ll}\text { References } & 24\end{array}$

\section{Introduction}

The goal of this paper is to study the behavior of conformally simplectic (contractive without loss of generality) mappings and flows in the neighborhood of an invariant torus, where the motion is equivalent to a rotation. In particular, we consider rotational Lagrangian tori; the corresponding quasi-periodic solutions might be associated to a transitive torus of maximal dimension (whose solution corresponding to a non-resonant - i.e., irrational, eventually Diophantine - frequency vector fills densely the torus) or a periodic torus (associated to a resonant frequency vector). These conformal systems appear in mechanical systems affected by a friction proportional to the velocity (a very common modeling assumption). The persistence of quasi-periodic solutions in conformally symplectic systems has been studied extensively. For our purposes, the most relevant reference is [7], since we will use some of the results there and we adopt the geometric point of view of that paper.

In this paper we show that, given a quasi-periodic solution, there is a neighborhood in which the motion is conjugate to a very simple motion, namely a rotation in the angles and a constant contraction in the actions (see Theorem 2 for a precise formulation).

In particular we show that all the maximal invariant tori (i.e., invariant tori with as many frequencies as the number of degrees of freedom) of these conformally symplectic systems are local attractors (or repellors). 
We would like to emphasize that the results of this paper are non-perturbative, they apply to systems that are far from integrable and without any Diophantine condition on the rotation. We just need to assume that a quasi-periodic solution exists. Of course, the existence of quasi-periodic solutions can be established by a variety of methods, some of which use Diophantine properties and proximity to integrable.

The method of proof starts by showing that a similar result is true infinitesimally. This is the phenomenon of automatic reducibility observed in [7] which shows that, given a quasi-periodic solution, there is a system of coordinates around the torus that makes the linearized evolution to be just constant coefficients. Hence, in a small neighborhood, we can get that the system (being close to the linearization) is close to a linear contraction in the actions and a rotation.

Once we have that the system is close to a constant skew-product, we use a method similar to the proof in [26] of Poincaré's linearization theorem [24]. One small wrinkle is that we need to use a deformation method as in $[22,27]$ to ensure that the change of variables giving the linearization is symplectic.

In Section 4.2.1 we show how these arguments can be transformed into algorithms. This also leads to some theoretical consequences such as 1) finite differentiable results, 2) relation with the theory of isochrones and perturbative expansion of the solution, 3) an alternative proof of the convergence based on the majorant method.

Moreover, we also show that the results obtained here also apply to quasi-periodic perturbations of systems with dissipation, which have been considered in the literature $([5,13,14])$. However, our method does not require that the system is analytic or close to the integrable case (see Section 5).

\section{Definitions and statement of the Results}

In this section we provide the definitions of conformally symplectic systems and of invariant tori; then we state the main result about a local model in the neighborhood of an invariant attractor.

2.1. Conformally symplectic systems. Let $\mathcal{M}$ be a symplectic manifold of dimension $2 n$, endowed with the standard scalar product and a symplectic form $\Omega$. We say that $f: \mathcal{M} \rightarrow \mathcal{M}$ is a conformally symplectic diffeomorphism when

$$
f^{*} \Omega=\lambda \Omega
$$


for some $\lambda$ real. Indeed, one can also assume that $\lambda$ is a function, say $\lambda: \mathcal{M} \rightarrow \mathbb{R}$, but we will always consider the case of $\lambda$ constant. For continuous systems we say that the flow $f_{t}: \mathcal{M} \rightarrow \mathcal{M}$ is a conformally symplectic flow, when

$$
f_{t}^{*} \Omega=e^{\eta t} \Omega
$$

for some $\eta$ real.

Remark 1. When $n=1$, taking as $\Omega$ the area form, any mapping admits a non-constant function $\lambda$. In these circumstances, there are results on linearization in neighborhoods, but they require extra assumptions and they involve other considerations.

We will not consider the case of non-constant $\lambda$ here, which can only happen when $n=1$; as it is well known ([3]), when $n \geq 2$ and $\mathcal{M}$ is connected, conformally symplectic systems occur only when $\lambda$ is a constant function.

In [3] there are even more general definitions that are also called "conformally symplectic". In this paper, however, we will stick to the definitions (2.1), (2.2).

We note that conformally symplectic flows are generated by conformally symplectic vector fields. Taking derivatives of (2.2) with respect to time, using the definition of the generator vector field $\mathcal{F}_{t}$ such that $\frac{d}{d t} f_{t}=\mathcal{F}_{t} \circ f_{t}$, we obtain:

$$
\left(f_{t}\right)^{*}\left(i_{\mathcal{F}_{t}} d \Omega+d\left(i_{\mathcal{F}_{t}} \Omega\right)\right)=\eta e^{\eta t} \Omega
$$

where $i_{\mathcal{F}_{t}}$ denotes the contraction with the vector field $\mathcal{F}_{t}$. Using $d \Omega=0$ and $(2.2)$, we obtain

$$
d\left(i_{\mathcal{F}_{t}} \Omega\right)=\eta \Omega
$$

If furthermore $\Omega=d \alpha$, then (2.3) becomes $d\left(i_{\mathcal{F}_{t}} \Omega\right)=d(\eta \alpha)$. Hence, $i_{\mathcal{F}_{t}} \Omega=\eta \alpha+G$, for some function $G$ with $d G=0$. If we write $G=d H$, we obtain

$$
i_{\mathcal{F}_{t}} \Omega=\eta \alpha+d H
$$

the vector fields satisfying (2.4) are called "exact conformally symplectic" fields. Clearly, all exact conformally symplectic fields are conformally symplectic. The converse is not necessarily true. Similarly, a diffeomorphism $f$ is exact conformally symplectic, whenever there exists a single-valued function $P$ such that

$$
f^{*} \alpha=\lambda \alpha+d P \text {. }
$$

However, in this paper exactness will not play an important role.

We will henceforth assume $|\lambda|<1$ or $\eta<0$. The complementary cases $|\lambda|>1$ and $\eta>0$ can be obtained by considering $f^{-1}$ and $f_{-t}$. We will not consider the case $|\lambda|=1$ 
(the symplectic case); indeed, the results obtained in this paper are not true for $|\lambda|=1$ (it is well known that the coefficients of the Birkhoff normal forms near an invariant torus are symplectic invariants). A very thorough discussion of Birkhoff normal forms near tori in symplectic maps can be found in [15].

2.2. Invariant tori. Given $\omega \in \mathbb{R}^{n}$, we denote by $T_{\omega}: \mathbb{T}^{n} \rightarrow \mathbb{T}^{n}$ the shift

$$
T_{\omega}(\theta)=\theta+\omega \text {. }
$$

We say that an embedding $K: \mathbb{T}^{n} \rightarrow \mathcal{M}(\mathcal{M}$ of dimension $2 n$ ) defines a rotational invariant torus for $f$, whenever the following invariance equation is satisfied:

$$
f \circ K=K \circ T_{\omega} .
$$

Similarly for flows the embedding $K$ defines a rotational invariant torus when

$$
f_{t} \circ K=K \circ T_{\omega t} .
$$

Note that if $K$ is an embedding, then $\mathcal{K}=K\left(\mathbb{T}^{n}\right)$ is a diffeomorphic copy of the torus.

2.2.1. Lagrangian properties of rotational tori in conformally symplectic mappings. We say that a parameterized torus of dimension $n$ is Lagrangian if

$$
K^{*} \Omega=0
$$

which in coordinates is equivalent to

$$
D K^{T}(\theta) J \circ K(\theta) D K(\theta)=0 .
$$

We remark that tori that satisfy (2.7) are called isotropic in symplectic geometry. When the dimension of the torus is half of the dimension of the phase space, they are called Lagrangian.

We will now show in detail that all rotational invariant tori (of any dimension) are isotropic. The reason why rotational tori are isotropic is that

$$
\begin{aligned}
& (f \circ K)^{*} \Omega=K^{*} f^{*} \Omega=\lambda K^{*} \Omega \\
& \left(K \circ T_{\omega}\right)^{*} \Omega=T_{\omega}^{*} K^{*} \Omega .
\end{aligned}
$$

If the torus is a rotational torus, both items in the formula above are equal. Writing $A(\theta) \equiv D K^{T}(\theta) J \circ K(\theta) D K(\theta)\left(A\right.$ is the matrix representation of $\left.K^{*} \Omega\right)$, we obtain

$$
\lambda A(\theta)=A(\theta+\omega) .
$$

Hence, $\sup _{\theta}|A(\theta)|=|\lambda| \sup _{\theta}|A(\theta)|$; since $|\lambda| \neq 1$, it is easy to show that $A \equiv 0$.

We call attention to the fact that the argument above is much simpler than the better known argument for symplectic maps, where, in order to conclude that that torus is 
symplectic, one needs to assume that the rotation is not resonant (i.e. $\omega \cdot k \notin \mathbb{Z}$ for all $\left.k \in \mathbb{Z}^{n}-\{0\}\right)$ and that the mapping is exact symplectic. In the conformally symplectic case, we do not need either of these assumptions.

Of course, when the frequency is not resonant, the motion on the torus is minimal (i.e. all the orbits are dense). When there are some resonances, the torus consists of a family of invariant tori each with minimal dynamics.

We emphasize that the results in this paper remain valid even in the case of resonant tori. We just need that the torus is Lagrangian, which in our case is ensured by the fact that the dimension of the torus is the same as the number of degrees of freedom.

2.3. Local models near an invariant torus. When considering the neighborhood of an invariant torus, we can assume, without loss of generality, that the manifold $\mathcal{M}$ coincides with a manifold $\mathcal{M}_{0}$ defined as

$$
\mathcal{M}_{0} \equiv \mathbb{T}^{n} \times B
$$

where $B$ is a ball of $\mathbb{R}^{n}$ around zero. An important property of $\mathcal{M}_{0}$ is that it is an Euclidean manifold, so that we can add vectors based at different points, compute averages and perform operations that are useful for analysis, even if geometrically unnatural (and, hence, not possible in arbitrary manifolds). Another important property of $\mathcal{M}_{0}$ is that it has natural complex extensions, so that it will be easy to deal with analytic functions. We consider $\mathcal{M}_{0}$ endowed with the Euclidean scalar product $\langle$,$\rangle and we denote by J$ the matrix corresponding to the symplectic matrix, namely if $u, v \in T_{x} \mathcal{M}$, then one has

$$
\Omega(u, v)=\langle J(x) u, v\rangle \text {. }
$$

We note that in $\mathcal{M}_{0}$ there is a standard symplectic form

$$
\Omega_{0}=\sum_{i=1}^{n} d A_{i} \wedge d \theta_{i},
$$

where $A_{i}$ are the coordinates in $B \subset \mathbb{R}^{n}$ and $\theta_{i}$ are the coordinates on $\mathbb{T}^{n}$.

An important example of a conformally symplectic mapping with respect to $\Omega_{0}$ is given by the mapping

$$
f_{0}(A, \theta)=(\lambda A, \theta+\omega)
$$

and similarly for flows we introduce

$$
f_{t}^{0}(A, \theta)=\left(e^{\eta t} A, \theta+\omega t\right) .
$$

Notice that (2.10) and (2.11) provide a constant contraction in the actions and a rotation by $\omega$ in the angles. For $\rho>0$ we introduce the following complex domains of a torus $\mathbb{T}^{n}$, 
of a set $B$ and of a manifold $\mathcal{M}$ :

$$
\begin{aligned}
\mathbb{T}_{\rho}^{n} & \equiv\left\{z=x+i y \in \mathbb{C}^{n} / \mathbb{Z}^{n}: x \in \mathbb{T}^{n},\left|y_{j}\right| \leq \rho, j=1, \ldots, n\right\} \\
B_{\rho} & \equiv\left\{z=x+i y \in \mathbb{C}^{n}: x \in B, \quad\left|y_{j}\right| \leq \rho, j=1, \ldots, n\right\} \\
\mathcal{M}_{\rho} & =\mathbb{T}_{\rho}^{n} \times B_{\rho} .
\end{aligned}
$$

For analytic functions in a domain $\mathcal{M}_{\rho}$ which extend continuously to the closure, we introduce the standard norm

$$
\|f\|_{\mathcal{M}_{\rho}}=\sup _{z \in \mathcal{M}_{\rho}}|f(z)|
$$

similarly for functions with more components. It is standard that the space of analytic functions in a domain extending to continuous functions in the boundary is a Banach space endowed with this norm.

Having fixed the notations, we state the main result concerning the conjugation to a rotation and a contraction near a rotational, Lagrangian invariant torus.

Theorem 2. Let $(\mathcal{M}, \Omega)$ be a $2 n$-dimensional analytic symplectic manifold, $f$ an analytic conformally symplectic contractive diffeomorphism as in (2.1) (respectively, $f_{t}$ be an analytic conformally symplectic flow as in (2.2)). Let $\omega \in \mathbb{R}^{n}$ and assume that there exists an analytic embedding $K: \mathbb{T}^{n} \rightarrow \mathcal{M}$, such that (2.5) (respectively, (2.6)) and (2.8) are satisfied. Then, there exists $\rho>0$ and an analytic diffeomorphism $g$ defined from $\mathcal{M}_{\rho}$ to a neighborhood of $\mathcal{K}=K\left(\mathbb{T}^{n}\right)$ in such a way that:

$$
\begin{aligned}
g^{-1} \circ f \circ g & =f_{0} \\
g^{*} \Omega_{0} & =\Omega \\
g(\theta, 0) & =K(\theta),
\end{aligned}
$$

where $f_{0}$ is given in (2.10), $\Omega_{0}$ is given in (2.9) and, respectively, for flows we have

$$
\begin{aligned}
g^{-1} \circ f_{t} \circ g & =f_{t}^{0} \\
g^{*} \Omega_{0} & =e^{\eta t} \Omega \\
g(\theta, 0) & =K(\theta),
\end{aligned}
$$

where $f_{t}^{0}, \eta$ are given in (2.11).

We remark that in Theorem 2 we do not impose any non-resonance condition on $\omega$; Theorem 2 applies even to $\omega \in \mathbb{Q}^{n}$. There exist similar results for finite differentiable mappings, which will be discussed and proved in Section 4.1. We notice that Theorem 2 implies that quasi-periodic solutions are local attractors. 
Remark 3. Notice that the solutions of the first equation in (2.13) are always non unique in the following sense. Assume that $g$ solves

$$
f \circ g=g \circ f_{0}
$$

and define $A_{\sigma, \mu}(\theta, s) \equiv(\theta+\sigma, \mu s)$ for $\sigma \in \mathbb{T}^{n}, \mu \in \mathbb{R}$. Note that, with this notation, $f_{0}=A_{\omega, \lambda}$.

We note that these mappings A commute. Hence, we have:

$$
f \circ\left(g \circ A_{\sigma, \mu}\right)=g \circ f_{0} \circ A_{\sigma, \mu}=\left(g \circ A_{\sigma, \mu}\right) \circ f_{0},
$$

namely, if $g$ solves (2.14), so does $\tilde{g} \equiv g \circ A_{\sigma, \mu}$ for any $\sigma \in \mathbb{T}^{n}, \mu \in \mathbb{R} \backslash\{0\}$; moreover, $\tilde{g}$ is a diffeomorphism. This non-uniqueness can be understood by saying that we can choose the origin of the angle variables and the scale of the linear variables.

We will show later, at the end of Section 4.2.1 that, when $\omega$ is an irrational translation, this is the only non-uniqueness of (2.14). The second condition of $(2.13), g^{*} \Omega_{0}=\Omega$, fixes the parameter $\mu$, while the origin of the phase remains arbitrary.

\section{Proof of Theorem 2}

The proof of Theorem 2 starts with the observation that the automatic reducibility introduced in [7] provides an approximate solution of (2.13) (i.e., a solution up to $O\left(A^{2}\right)$ ). The second step consists in using a deformation method as in [22], [4] to modify the first approximate conjugation (which is not necessarily symplectic), so that it is also symplectic (the modification has to be done only to the high order terms to ensure that it is still an approximate solution). After that, there are several standard methods to show that we can use this approximate solution to construct a true solution.

In this section, we have chosen to present a method based on the pioneering work described in [26]. It is interesting to note that, because of the conformal symplectic properties, the map behaves very much like a one-dimensional map. Another standard method, which could be useful in more complicated situations, is the deformation method described in [4]. As we will see later, this method works also for $C^{r}$ systems, $r \in \mathbb{N}$, or in $C^{\infty}$. Later, in Section 4.3 we will present a method based on the classic method of majorants, which only works for analytic systems. The method of majorants was the main tool available at the time of [24].

We note that the second step (getting the symplectic form to be $\Omega_{0}$ ) can be omitted at the only price that we do not require that the symplectic form coincides with $\Omega_{0}$. 
We provide the proof of Theorem 2 for mappings; the proof for flows is a straightforward extension of that for mappings and it is left to the reader.

\subsection{A system of coordinates and an approximate solution of (2.13) in a neigh-} borhood of the torus. The goal of this section is to produce an approximate solution of (2.13), see Lemma 5. This will be an almost immediate consequence of the following Lemma 4 (see Section 3 of [7]), that we will use in a neighborhood of the torus.

Lemma 4. Let $\mathcal{M}$ be a symplectic manifold and let $f$ be an analytic conformally symplectic manifold with symplectic form $\Omega$, such that $f^{*} \Omega=\lambda \Omega$ with $\lambda \in \mathbb{R},|\lambda|<1$. Assume that $K: \mathbb{T}^{n} \rightarrow \mathcal{M}$ is an analytic embedding, that $\omega \in \mathbb{R}^{n}$ is such that (2.5) holds and that $K\left(\mathbb{T}^{n}\right)$ is a Lagrangian manifold.

Let us define the quantity

$$
N(\theta)=\left(D K(\theta)^{T} D K(\theta)\right)^{-1}
$$

and let

$$
\tilde{M}(\theta)=M(\theta)\left(\begin{array}{cc}
\operatorname{Id} & B(\theta) \\
0 & \operatorname{Id}
\end{array}\right)
$$

where

$$
M(\theta)=\left[D K(\theta) \mid J^{-1} \circ K(\theta) D K(\theta) N(\theta)\right] .
$$

Let $B$ satisfy the equation

$$
B(\theta)+S(\theta)-\lambda B(\theta+\omega)=0,
$$

where

$$
\begin{aligned}
P(\theta) & =D K(\theta) N(\theta) \\
\gamma(\theta) & =D K(\theta)^{T} J^{-1} \circ K(\theta) D K(\theta) \\
S(\theta) & =P(\theta+\omega)^{T} D f \circ K(\theta) J^{-1} \circ K(\theta) P(\theta)-\lambda N(\theta+\omega)^{T} \gamma(\theta+\omega) N(\theta+\omega) .
\end{aligned}
$$

Then, one has

$$
\begin{gathered}
D f \circ K(\theta) \tilde{M}(\theta)=\tilde{M}(\theta+\omega)\left(\begin{array}{cc}
\mathrm{Id} & 0 \\
0 & \lambda \mathrm{Id}
\end{array}\right), \\
M^{T} J \circ K M=J_{0},
\end{gathered}
$$

where

$$
J_{0}=\left(\begin{array}{cc}
0 & \mathrm{Id} \\
-\mathrm{Id} & 0
\end{array}\right)
$$


Proof. Let $M$ and $N$ be as in (3.3), (3.1). According to [7], one has that

$$
D f \circ K(\theta) M(\theta)=M(\theta+\omega)\left(\begin{array}{cc}
\operatorname{Id} & S(\theta) \\
0 & \lambda \mathrm{Id}
\end{array}\right) .
$$

From this relation we obtain:

$$
\begin{aligned}
D f \circ K(\theta) \tilde{M}(\theta) & =M(\theta+\omega)\left(\begin{array}{cc}
\operatorname{Id} & S(\theta) \\
0 & \lambda \operatorname{Id}
\end{array}\right)\left(\begin{array}{cc}
\operatorname{Id} & B(\theta) \\
0 & \operatorname{Id}
\end{array}\right) \\
& =\tilde{M}(\theta+\omega)\left(\begin{array}{cc}
\operatorname{Id} & -B(\theta+\omega) \\
0 & \operatorname{Id}
\end{array}\right)\left(\begin{array}{cc}
\operatorname{Id} & S(\theta) \\
0 & \lambda \operatorname{Id}
\end{array}\right)\left(\begin{array}{cc}
\operatorname{Id} & B(\theta) \\
0 & \operatorname{Id}
\end{array}\right) \\
& =\tilde{M}(\theta+\omega)\left(\begin{array}{cc}
\operatorname{Id} & -\lambda B(\theta+\omega)+S(\theta)+B(\theta) \\
0 & \lambda \operatorname{Id}
\end{array}\right) .
\end{aligned}
$$

Defining $B(\theta)$ such that (3.4) is satisfied, then we obtain (3.5). Notice that (3.4) does not involve zero divisors for any $\omega \in \mathbb{R}^{n}$ and that it can be solved whenever $|\lambda| \neq 1$; on the contrary, if $|\lambda|=1$ there appear zero divisors and one needs to impose a constraint on the frequency. Indeed, if $|\lambda|<1$, an explicit solution of (3.4) is given by

$$
B(\theta)=\sum_{j \geq 0} \lambda^{j} S(\theta+j \omega)
$$

Note that the formula (3.8) allows us to estimate the norm of the solution for any translation invariant norm (e.g., the analytic norms introduced in (2.12) or the $C^{r}$ norm):

$$
\|B\| \leq \sum_{j \geq 0} \lambda^{j}\left\|S \circ T_{j \omega}\right\|=(1-\lambda)^{-1}\|S\| .
$$

This estimate will be used later in order to prove the convergence of the series. We conclude by stating that (3.6) is a consequence of (3.1), (3.3), (3.7) and (2.8).

Note that $\tilde{M}(\theta)$ is an analytic matrix valued function. The geometric meaning of Lemma 4 is that around the points in $\mathcal{K}=K\left(\mathbb{T}^{n}\right)$, we can find a frame of vectors such that when we express a vector in this frame, the action of the derivative is just a multiplication by the matrix $\left(\begin{array}{cc}\mathrm{Id} & 0 \\ 0 & \lambda \mathrm{Id}\end{array}\right)$. The vectors which get transported by $D f$ are precisely the vectors in the tangent space to $\mathcal{K}$. The existence of this frame is a requirement considerably stronger than the assumptions in $[11,12]$, so that we will be able to obtain stronger conclusions than those drawn in $[11,12,19]$. The following lemma provides an approximate solution in the neighborhood of the invariant torus.

Lemma 5. With the notations of Lemma 4, consider the analytic diffeomorphism $h$ : $\mathcal{M}_{0} \rightarrow \mathcal{M}$, defined by

$$
h(\theta, s)=K(\theta)+[\tilde{M}(\theta)]_{2} s,
$$


where $[\tilde{M}(\theta)]_{2}$ denotes the second column of $\tilde{M}(\theta)$ in the block decomposition of (3.2). Then, one has:

$$
\begin{aligned}
f \circ h(\theta, 0) & =h(\theta+\omega, 0) \\
D f \circ h(\theta, 0) D_{\theta} h(\theta, 0) & =D_{\theta} h(\theta+\omega, 0) \\
h^{*} \Omega & =\Omega_{0}+O\left(s^{2}\right) .
\end{aligned}
$$

Proof. We note that for $|s|$ sufficiently small, it is clear that $h$ is a diffeomorphism into the range, which is a consequence of the implicit function theorem, because

$$
\begin{aligned}
& D_{\theta} h(\theta, 0)=D K(\theta) \\
& D_{s} h(\theta, 0)=[\tilde{M}(\theta)]_{2} .
\end{aligned}
$$

The relations (3.9) are a consequence of (2.5), (3.5) and (3.10). In particular, the last relation is true because $D h(\theta, 0)=M(\theta)$ and we have (3.6).

3.2. Making the approximate conjugation symplectic. The goal of this section is to prove the following Lemma 6, which is a simple consequence of Moser's deformation technique $([22])$.

Lemma 6. Let $\tilde{\Omega}$ be an analytic symplectic form, such that $\left|\left(\tilde{\Omega}-\Omega_{0}\right)\right|(\theta, s)=O\left(|s|^{2}\right)$. Then, there exists an analytic diffeomorphism $k$, defined as

$$
k(\theta, s)=(\theta, s)+O\left(|s|^{2}\right)
$$

in such a way that

$$
\left(k^{-1}\right)^{*} \tilde{\Omega}=\Omega_{0}
$$

The proof of Lemma 6 follows by just going over the procedure of the deformation method of the global Darboux theorem (see [22], [27]). The goal is to check that we preserve the analyticity and the fact that (3.11) holds.

Proof. Following the deformation method of [22], [27], we set

$$
\Omega_{\varepsilon}=\varepsilon \tilde{\Omega}+(1-\varepsilon) \Omega_{0}, \quad 0 \leq \varepsilon \leq 1 .
$$

We note that $\Omega_{\varepsilon}=\Omega_{0}+O\left(|s|^{2}\right)$ uniformly in $\varepsilon$ and, since the non-degeneracy is an open condition, then $\Omega_{\varepsilon}$ is non degenerate for small $s$ uniformly in $\varepsilon$. Note also that $d \Omega_{\varepsilon}=\varepsilon d \tilde{\Omega}+(1-\varepsilon) d \Omega_{0}=0$.

We now try to find a family of diffeomorphisms $k_{\varepsilon}$ such that

$$
k_{\varepsilon}^{*} \Omega_{\varepsilon}=\Omega_{0}, \quad k_{0}=\mathrm{Id} .
$$


We see that if we write

$$
\frac{d}{d \varepsilon} k_{\varepsilon}=\mathcal{F}_{\varepsilon} \circ k_{\varepsilon}
$$

for a generator vector field $\mathcal{F}_{\varepsilon}$, then using Cartan's magic formula (3.12) is equivalent to

which provides

$$
d\left(i_{\mathcal{F}_{\varepsilon}} \Omega_{\varepsilon}\right)+i_{\mathcal{F}_{\varepsilon}} d \Omega_{\varepsilon}+\frac{d}{d \varepsilon} \Omega_{\varepsilon}=0
$$

$$
d\left(i_{\mathcal{F}_{\varepsilon}} \Omega_{\varepsilon}\right)+\left(\tilde{\Omega}-\Omega_{0}\right)=0 .
$$

Since $\Omega_{\varepsilon}$ is non degenerate and $\Omega_{\varepsilon}-\Omega_{0}=O\left(|s|^{2}\right)$, we obtain that we can find a unique $\mathcal{F}_{\varepsilon}$, defined for $|s| \leq s_{0}$ for some $s_{0} \ll 1$, satisfying (3.13). This function $\mathcal{F}_{\varepsilon}$ is uniformly analytic in $\varepsilon$, namely $\mathcal{F}_{\varepsilon}$ is analytic on $\mathbb{T}_{\rho}^{n} \times B_{\rho}$ and there it is uniformly bounded in $\varepsilon$. Moreover, we have

$$
\left|\mathcal{F}_{\varepsilon}(\theta, s)\right| \leq C|s|^{2} \quad \forall \theta \in \mathbb{T}^{n}
$$

By Gronwall's inequality we conclude that $k_{1}$ is defined in a small neighborhood of the torus and it satisfies:

$$
\left|k_{1}(\theta, s)-(\theta, s)\right| \leq C|s|^{2} \quad \forall \theta \in \mathbb{T}^{n} .
$$

Of course, $k_{1}$ is also analytic since it is the solution of an analytic differential equation.

If we apply Lemma 6 to the case $\tilde{\Omega}=\left(h^{-1}\right)^{*} \Omega$, where $h$ is the local diffeomorphism produced in Lemma 5 , we obtain a diffeomorphism $k$. Then, one has that $\tilde{h}=k \circ h$ satisfies

$$
\begin{aligned}
\tilde{h}^{-1} \circ f \circ \tilde{h} & =f_{0}+O\left(|s|^{2}\right) \\
\left(\tilde{h}^{-1}\right)^{*} \Omega & =\Omega_{0} \\
\left(\tilde{h}^{-1} \circ f \circ \tilde{h}\right)^{*} \Omega_{0} & =\lambda \Omega_{0} .
\end{aligned}
$$

The first line in (3.14) is proven as follows. Let us first remark that for any $v=v(\theta)$, as a consequence of (3.5) one has that

$$
D f \circ K(\theta) v(\theta)=\lambda v(\theta+\omega) .
$$

Then, we prove that

$$
f \circ \tilde{h}=\tilde{h} \circ f_{0}+O\left(|s|^{2}\right) .
$$

In fact, expanding the left hand side to the second order, one has

$$
f \circ \tilde{h}(\theta, 0)+D f \circ \tilde{h}(\theta, 0) D_{s} \tilde{h}(\theta, 0) s=\tilde{h}(\theta, s) \circ f_{0}+O\left(|s|^{2}\right),
$$

which, using Lemma 5, corresponds to

$$
f \circ K(\theta)+D f \circ K(\theta)[\tilde{M}(\theta)]_{2} s=K(\theta+\omega)+[\tilde{M}(\theta+\omega)]_{2} \lambda s+O\left(|s|^{2}\right)
$$


which is true due to (2.5) and (3.15).

The second of (3.14) comes from applying Lemma 6 to $\tilde{\Omega}$, while the third relation comes from the first of (3.14).

3.3. End of the proof of Theorem 2. In this section we complete the proof of Theorem 2. As it turns out, the method that was used in [26] for one-dimensional mappings allows to prove our result. Let

$$
\tilde{f}=\tilde{h}^{-1} \circ f \circ \tilde{h} .
$$

Remember that now we have two mappings in $\mathcal{M}_{0}$, namely $\tilde{f}$ and $f_{0}$, which are defined in (3.16) and (2.10), respectively. Both of them are $\lambda$-conformal for $\Omega_{0}$ and one has

$$
\left|f_{0}(\theta, s)-\tilde{f}(\theta, s)\right|=O\left(|s|^{2}\right) \quad \forall \theta \in \mathbb{T}^{n} .
$$

Since $\tilde{f}$ is obtained from $f$ through analytic changes of variables, which also preserve the geometry, Theorem 2 will be established as soon as we produce an analytic symplectic mapping that conjugates $\tilde{f}$ and $f_{0}$ in a neighborhood of $\{s=0\}$ on $\mathcal{M}_{0}$. Both $\tilde{f}, f_{0}$ are analytic and we can consider their extensions to $\mathcal{M}_{\rho}$.

Following [26] we consider

$$
g_{j}=f_{0}^{-j} \circ \tilde{f}^{j}
$$

Note that

$$
f_{0} \circ g_{j}=f_{0}^{-(j-1)} \circ \tilde{f}^{j-1} \circ \tilde{f}=g_{j-1} \circ \tilde{f} .
$$

Furthermore, since both $\tilde{f}, f_{0}$ are $\lambda$-conformally symplectic for $\Omega_{0}$, then $g_{j}$ is symplectic for $\Omega_{0}$ and we also have

$$
\begin{aligned}
g_{j}(\{s=0\}) & =\{s=0\} \\
\left.D g_{j}\right|_{\{s=0\}} & =\mathrm{Id} .
\end{aligned}
$$

Hence, as soon as we show that $g_{j}$ converges uniformly in $\mathcal{M}_{\rho}$ to $g$ (for some $\rho>0$ ), it will follow that the limit $g$ satisfies that $g$ is symplectic for $\Omega_{0}$ and that the following relation holds true:

$$
|g(\theta, s)-(\theta, s)|=O\left(|s|^{2}\right) \quad \forall \theta \in \mathbb{T}^{n} .
$$

We notice that the argument for the convergence of $g_{j}$ is very similar to Cook's method in scattering theory (see [23] for developments of the analogy between scattering theory and equivalence of dynamical systems). Then we have:

$$
\begin{aligned}
\left\|g_{j-1}-g_{j}\right\|_{\mathcal{M}_{\rho}} & =\left\|f_{0}^{-(j-1)} \circ \tilde{f}^{j-1}-f_{0}^{-(j-1)} \circ\left(f_{0}\right)^{-1} \circ \tilde{f} \circ \tilde{f}^{j-1}\right\|_{\mathcal{M}_{\rho}} \\
& \leq\left\|f_{0}^{-(j-1)}-f_{0}^{-(j-1)} \circ\left(f_{0}\right)^{-1} \circ \tilde{f}\right\|_{\tilde{f}^{j-1}\left(\mathcal{M}_{\rho}\right)} .
\end{aligned}
$$


We now observe that

$$
\tilde{f}^{j-1} \mathcal{M}_{\rho} \subset \mathcal{M}_{(\lambda+\varepsilon)^{j-1} \rho}
$$

where $\varepsilon=\varepsilon(\rho)$ decreases to zero as $\rho$ tends to zero. Since $f_{0}$ and $\tilde{f}$ are tangent, we have that

$$
\left\|f_{0}^{-1} \circ \tilde{f}-\operatorname{Id}\right\|_{\mathcal{M}_{(\lambda+\varepsilon)^{j-1} \rho}} \leq C(\lambda+\varepsilon)^{2(j-1)} \rho,
$$

for some constant $C>0$.

By the mean value theorem, since $\operatorname{Lip}\left(f_{0}^{-1}\right) \leq \lambda^{-1}$, we have

$$
\left\|g_{j-1}-g_{j}\right\|_{\mathcal{M}_{\rho}} \leq C \lambda^{-(j-1)}(\lambda+\varepsilon)^{2(j-1)} \rho
$$

The requirement that $|\lambda+\varepsilon|<1$ can be met by assuming that $\rho$ is sufficiently small.

The sum of the above terms provides a geometric series which is absolutely convergent (recall that $|\lambda|<1$ and $\varepsilon$ is arbitrarily small); this concludes the proof of Theorem 2 . Note also that the above argument shows that the $g_{j}$ 's also send bounded domains into bounded domains.

\section{Some Complements And extensions of the Proof}

As it can be seen from the previous sections, the method of proof is very flexible and it can lead to several other results with minor modifications, as we are going to describe below.

4.1. Finite differentiable and $C^{\infty}$ results. Notice that the first two steps of the proof (the automatic reducibility and the deformation to obtain the symplectic nature of the conjugacy) require only that the maps have two derivatives. The conjugating maps thus obtained loose two derivatives with respect to the original map.

As for the last step of the proof of Theorem 2, namely the scattering method described in Section 3.3, it is also valid for finite differentiable maps or for $C^{\infty}$ maps (the latter case was considered in [26]). In fact, we show now that an inequality of the type (3.17) holds whenever the norm is computed over $C^{r}(\mathcal{M})$ for some $r>0$, instead than over the space of analytic functions in $\mathcal{M}_{\rho}$.

Since $f_{0}$ is an affine function, it is clear that $\left\|D f_{0}^{-j}\right\|_{C^{0}}=\lambda^{-j}$ and $D^{k} f_{0}^{-j}=0$ for $k \geq 2$. Using the above relations and that $f_{0}$ is affine, we get:

$$
\left\|g_{j}-g_{j-1}\right\|_{C^{r}(\mathcal{M})} \leq C \lambda^{-(j-1)}\left\|\left(f_{0}^{-1} \circ \tilde{f}-\mathrm{Id}\right) \circ \tilde{f}^{j-1}\right\|_{C^{r}(\mathcal{M})}
$$


Here and in what follows, we denote by $C$ terms that are independent of $j$. To estimate the expression above, we use Faa-Di-Bruno formula [2, 10, 1], as well as estimates for derivatives of highly iterated functions [9, p. 574],[4].

We denote by $\gamma \equiv f_{0}^{-1} \circ \tilde{f}-$ Id; by Faa-di-Bruno formula, we have:

$$
\begin{aligned}
& D^{k}\left(\gamma \circ \tilde{f}^{j-1}\right) \\
& \quad=\sum_{m_{1}+2 m_{2}+\cdots k m_{k}=k} C\left(k, m_{1}, \cdots m_{k}\right)\left(D^{m_{1}+\cdots+m_{k}} \gamma\right) \circ \tilde{f}^{j-1}\left(D \tilde{f}^{j-1}\right)^{\otimes m_{1}} \cdots\left(D^{k} \tilde{f}^{j-1}\right)^{\otimes m_{k}},
\end{aligned}
$$

where $C\left(k, m_{1}, \cdots, m_{k}\right)=k ! /\left(m_{1} ! \cdots m_{k} !\right)$. Our goal is to show that if we estimate the $C^{0}$ norm of all the terms on the right hand side of (4.1), we obtain series that are summable in $j$ when multiplied by the factor $\lambda^{-(j-1)}$. In this way, we will obtain that $\lambda^{-(j-1)} D^{k}\left(\gamma \circ \tilde{f}^{j-1}\right)$ can be summed in $j$.

The estimates of highly iterated functions in $[9$, p. 574] and [4], give the following estimates (which are somewhat pessimistic):

$$
\left\|D^{l} \tilde{f}^{j-1}\right\|_{C^{0}(\mathcal{M})} \leq C_{l}(\lambda+\varepsilon)^{j-1}(j-1)^{l+1}, \quad l>0 .
$$

Hence, we can estimate the terms in the sum at the right hand side of (4.1) by

$$
\begin{aligned}
\left\|\left(D^{m_{1}+\cdots+m_{k}} \gamma\right) \circ \tilde{f}^{j-1}\left(D \tilde{f}^{j-1}\right)^{\otimes m_{1}} \cdots\left(D^{k} \tilde{f}^{j-1}\right)^{\otimes m_{k}}\right\|_{C^{0}(\mathcal{M})} \\
\leq C(\lambda+\varepsilon)^{(j-1) \sum_{i=1}^{k} m_{i}}(j-1)^{(k+1) \sum_{i=1}^{k} m_{i}} .
\end{aligned}
$$

Of course, the dominant term in (4.2) is the exponential factor, while the polynomial factor is subdominant. We note that since $\sum_{i=1}^{k} i m_{i}=k$, we have $\sum_{i=1}^{k} m_{i} \geq 2$ except in the case $m_{k}=1, m_{k-1}=m_{k-2}=\ldots=m_{1}=0$. This establishes easily that (4.2) is a series summable in $j$, except when $m_{k}=1, m_{k-1}=m_{k-2}=\ldots=m_{1}=0$. This latter case will require a more precise argument that we develop now.

When $m_{k}=1, m_{k-1}=m_{k-2}=\ldots=m_{1}=0$, we observe that (4.2) is too conservative. In this case, $\left\|\left(D^{m_{1}+\cdots+m_{k}} \gamma\right) \circ \tilde{f}^{j-1}\right\|_{C^{0}(\mathcal{M})}=\left\|(D \gamma) \circ \tilde{f}^{j-1}\right\|_{C^{0}(\mathcal{M})}$ and we can estimate

$$
\left\|(D \gamma) \circ \tilde{f}^{j-1}\right\|_{C^{0}(\mathcal{M})} \leq C(\lambda+\varepsilon)^{j-1},
$$

because, as we have argued, $\gamma$ vanishes up to second order and $f^{j-1}$ is exponentially close to the attractor. Hence, the $C^{0}$ norm of the term at the right hand side of (4.1) corresponding to $m_{k}=1, m_{k-1}=m_{k-2}=\ldots=m_{1}=0$, which is just $\left\|(D \gamma) \circ \tilde{f}^{j-1} D \tilde{f}^{j-1}\right\|_{C^{0}}$, can be estimated by $C(\lambda+\varepsilon)^{2(j-1)}(j-1)^{2}$, which gives rise to a convergent series in $j$ when multiplied by $(\lambda+\varepsilon)^{-(j-1)}$. 
Therefore, we see that

$$
\left\|\gamma \circ \tilde{f}^{j-1}\right\|_{C^{r}(\mathcal{M})} \leq C(\lambda+\varepsilon)^{2(j-1)}(j-1)^{2 r}
$$

(we are very wasteful in the polynomial terms, to simplify the expressions, since the exponential terms are subdominant and do not play any role) and, hence we obtain

$$
\left\|g_{j}-g_{j-1}\right\|_{C^{r}(\mathcal{M})} \leq C \lambda^{-(j-1)}(\lambda+\varepsilon)^{2(j-1)}(j-1)^{2 r}
$$

which, together with the convergence in $C^{0}$ - established in our study of the analytic case - shows that $g_{j}$ converges in $C^{r}$.

We note that the $\rho$ required in the above argument may depend on $r$. To establish the $C^{\infty}$ result, we observe, following [26], that the domain where the series $\sum_{j} \| g_{j}-$ $g_{j-1} \|_{C^{r}\left(\mathcal{M}_{\rho}\right)}$ converges is independent of $r$. Indeed, assume that we have the conjugacy in an open set $U_{r}$ around the torus. Using the invariance equation (2.13), we see that $g=f^{-1} \circ g \circ f_{0}$, so that we can extend the definition to $f_{0}^{-1}\left(U_{r}\right)$. The extended function is clearly as differentiable as $\left.g\right|_{U_{r}}$. Repeating the argument $n$ times, we obtain that we can define the function $g$ in $f_{0}^{-n}\left(U_{r}\right)$ and that it is $C^{r}$ there. Taking $n$ sufficiently large, we obtain $\mathcal{M}_{\rho} \subset f_{0}^{-n}\left(U_{r}\right)$.

4.2. Relation with the theory of isochrones. An important consequence of (2.13) is that the stable manifolds (in the sense of the theory of normally hyperbolic manifolds) admit a topological characterization.

In the theory of normally hyperbolic manifolds, the stable manifold at $K(\theta)$ is defined as

$$
W_{K(\theta)}^{s t} \equiv\left\{y \in \mathbb{R}^{n}: \quad\left|f^{n}(y)-f^{n}(K(\theta))\right| \leq C_{\varepsilon}(\lambda+\varepsilon)^{n}, \quad n>0, \quad 0<\varepsilon \ll 1\right\} .
$$

Note that for general normally hyperbolic manifolds, $W_{K(\theta)}^{s t}$ is not a topological invariant (it involves a rate of convergence, which is not a topological invariant).

In our case, we have the following equalities; in particular, note that the last one shows that, for our systems, $W_{K(\theta)}^{s t}$ has a topological characterization:

$$
\begin{aligned}
W_{K(\theta)}^{s t} & \equiv\left\{y \in \mathbb{R}^{n}: \quad\left|f^{n}(y)-f^{n}(K(\theta))\right| \leq C_{\varepsilon}(\lambda+\varepsilon)^{n}, \quad n>0, \quad 0<\varepsilon \ll 1\right\} \\
& =\left\{y \in \mathbb{R}^{n}: \quad\left|f^{n}(y)-f^{n}(K(\theta))\right| \leq C \lambda^{n}, \quad n>0\right\} \\
& =\left\{y \in \mathbb{R}^{n}: \quad \lambda^{-n}\left|f^{n}(y)-f^{n}(K(\theta))\right| \rightarrow C, \quad n>0\right\} \\
& =\left\{y \in \mathbb{R}^{n}: \quad\left|f^{n}(y)-f^{n}(K(\theta))\right| \rightarrow 0, \quad n \rightarrow+\infty\right\} .
\end{aligned}
$$

Note that (4.3) states that the set of points whose orbits converge to the orbit of $K(\theta)$ is the same as the set of points whose orbits converge exponentially fast with multiplier 
$\lambda$ to the orbit of $K(\theta)$. Furthermore, according to (4.3) the optimal exponential rate is reached (i.e., no polynomial corrections are needed) and indeed, the rate is optimal.

Remark 7. We note that a corollary of the above argument is that for all $v \in T_{x} W_{K(\theta)}^{s t}$ :

$$
\lim _{n \rightarrow \infty}\left|D f^{n}(x) v\right|^{1 / n}=\lambda,
$$

so that $\lambda$ is the Lyapunov number along the directions of the stable manifold.

There are, of course, other directions that have Lyapunov multiplier 1 and these are all the Lyapunov multipliers that appear in the system.

Let us denote by $W_{K(\theta)}^{s t, l o c}$ the intersection of the connected component of the stable manifold including the torus with a neighborhood of the torus (say $s \leq s_{0}$ for some $s_{0}$ small). Taking into account that

$$
f^{n}(g(\theta, s))=g\left(f_{0}^{n}(\theta, s)\right)=g\left(\theta+n \omega, \lambda^{n} s\right),
$$

we obtain that $W_{K(\theta)}^{\text {st,loc }}$ can be characterized as the image of vertical lines (i.e., holding $\theta$ fixed) under the map $g$ :

$$
W_{K(\theta)}^{s t, l o c}=\left\{g(\theta, s): \quad s \in \mathbb{R}^{n}\right\} .
$$

Of course, for general normally hyperbolic manifolds, there could be points whose orbits converge sub-exponentially (e.g. polynomially) or it could happen that there are no points which converge to the optimal exponential rate (e.g. they could converge as $\left.\lambda^{n} n^{p}\right)$.

The reason why (4.3), (4.4) are true is that a similar set of equivalences holds true for the map $f_{0}$ (this uses the fact that the map $f_{0}$ is the product of a rotation and a contraction). For $f_{0}$ the set of points whose orbit converges to the orbit of $\theta$ is precisely the points with the same $\theta$. The orbits of these points converge locally with the optimal exponential rate.

Once we know that this characterization is true for the map $f_{0}$, we observe that since $g$ is a diffeomorphism, it preserves not only the fact that orbits converge, but also the rates of convergence.

Remark 8. The geometric meaning of $W_{K(\theta)}^{s t}$ is the set of points which asymptotically have the same phase as the point $K(\theta)$. Therefore, for limit cycles these sets were named isochrones in [28], where their importance in biology is also presented. The relation of isochrones to stable manifolds was pointed out in [16]. Our results show that the concept of isochrones can also be extended to quasi-periodic solutions of conformally symplectic systems. 
4.2.1. Computational aspects. An important practical consequence of (4.3) is that $g$ satisfies some functional equations (2.13) that make its computation possible. Indeed, the case of limit cycles has been considered in [17, 20] and related algorithms have been presented in [21] for the symplectic case. We observe that, if we write

$$
g(\theta, s)=\sum_{\ell \in \mathbb{Z}_{+}} g_{\ell}(\theta) s^{\ell}
$$

and if we substitute this expansion in (2.13), equating terms of the same order in $s$, we obtain

$$
\begin{aligned}
& f \circ g_{0}=g_{0} \circ T_{\omega} \\
& D f \circ g_{0} g_{1}=\lambda g_{1} \circ T_{\omega} \\
& \ldots \\
& D f \circ g_{0} g_{\ell}=\lambda^{\ell} g_{\ell} \circ T_{\omega}+S_{\ell}, \quad \ell \geq 2,
\end{aligned}
$$

where $S_{\ell}$ is a polynomial expression in $g_{1}, \cdots, g_{\ell-1}$. It is a rather explicit expression, because it is obtained by applying the chain rule. In practice, each expression in (4.5) can be readily computed using the methods of automatic differentiation $([6],[18])$. Hence, one can consider (4.5) as a recursive set of equations. We can assume inductively that $g_{0}, g_{1}, \ldots g_{\ell-1}$ are known and then use the third equation of (4.5) to find $g_{\ell}$. As we will see, the equations for $\ell=0,1$ are special, but then there is a systematic unique solution for all $\ell \geq 2$. Notice also the similarity of this procedure with the Lindstedt series which appear in Celestial Mechanics. However, in contrast with the Lindstedt series, the estimate of our series is elementary, since they do not contain small divisors (for example, the classical majorant method suffices as we are going to describe below).

The first equation of (4.5) admits a solution showing that we can take $g_{0}$ to be the parameterization $K$ of an invariant torus. When $\omega$ is irrational, once we choose the torus to study, the only free choice is the origin of coordinates, so that there is uniqueness up to a translation. As indicated in the automatic reducibility procedure described in Lemma 4, the second equation of (4.5) admits the solution $g_{1}=J^{-1} \circ g_{0} D g_{0} N$; such solution is unique up to a multiple.

The equations for $\ell \geq 2$ are readily solvable as we will show. We note that using the automatic reducibility and writing $g_{\ell}=\tilde{M} u_{\ell}$ with $\tilde{M}$ as in (3.2), we obtain that the last equation in (4.5) becomes

$$
D f \circ g_{0} \tilde{M} u_{\ell}=\lambda^{\ell} \tilde{M} \circ T_{\omega} u_{\ell} \circ T_{\omega}+S_{\ell}
$$


where the only unknown is now $u_{\ell}$. Using (3.2) and (3.5), the equation (4.6) becomes

$$
\left(\begin{array}{cc}
\operatorname{Id} & 0 \\
0 & \lambda \mathrm{Id}
\end{array}\right) u_{\ell}-\lambda^{\ell} u_{\ell} \circ T_{\omega}=\tilde{M}^{-1} \circ T_{\omega} S_{\ell}
$$

We can write the equation (4.7) as a system of two equations using the component notation $u_{\ell}=\left(\begin{array}{l}\left(u_{\ell}\right)_{1} \\ \left(u_{\ell}\right)_{2}\end{array}\right)$. We obtain that $(4.7)$ is equivalent to

$$
\begin{aligned}
& \left(u_{\ell}\right)_{1}-\lambda^{\ell}\left(u_{\ell}\right)_{1} \circ T_{\omega}=\left((\tilde{M})^{-1} \circ T_{\omega} S_{\ell}\right)_{1} \\
& \lambda\left(u_{\ell}\right)_{2}-\lambda^{\ell}\left(u_{\ell}\right)_{2} \circ T_{\omega}=\left((\tilde{M})^{-1} \circ T_{\omega} S_{\ell}\right)_{2} .
\end{aligned}
$$

Note that the second equation of (4.8) is equivalent to

$$
\left(u_{\ell}\right)_{2}-\lambda^{\ell-1}\left(u_{\ell}\right)_{2} \circ T_{\omega}=\lambda^{-1}\left((\tilde{M})^{-1} \circ T_{\omega} S_{\ell}\right)_{2} .
$$

We see that for $\ell \geq 2$, both equations in (4.8) admit a unique solution similar to that in (3.8). Therefore, we see that, once we choose $u_{1}$ (and, of course, $u_{0}$ ), then all the coefficients $u_{\ell}, \ell \geq 2$, are uniquely determined. Furthermore, we have recursive bounds

$$
\left\|u_{\ell}\right\|_{\mathcal{M}_{\rho}} \leq C\left\|S_{\ell}\right\|_{\mathcal{M}_{\rho}} .
$$

We therefore, conclude that the only solutions of the conjugacy equation are obtained just by changing the origin in the equation or by scaling the linear coordinates. These are the non-uniqueness that we had already observed in Remark 3.

Remark 9. Notice that, as a corollary of the uniqueness, we obtain that for analytic systems all solutions of the conjugacy equation which have the appropriate scale factor are automatically symplectic. The reason is that we have established in Theorem 2 that there exists a symplectic solution; the solutions with a different scale factor cannot be symplectic. It is possible to give a geometric proof of this fact, which has the advantage that it applies also to finite differentiable maps. One can really understand this because we can construct the conjugacy also by $h=\lim _{j \rightarrow \infty} h_{j} \equiv \lim _{j \rightarrow \infty} f_{0}^{-j} \circ D\left(h \circ f^{j}\right) \circ f^{j}$. Imposing that Dh०f satisfies (3.6), we obtain $h_{j}^{*} \Omega=\Omega_{0}$ and hence the symplectic property.

4.3. Convergence of the series by the majorant method. For the sake of completeness, we present the proof of the convergence of the perturbative series developed in (4.5) using the method of majorants; this was the method of proof that was used in [24] (see also [25]). It is a general method to show convergence of formal solutions of functional equations. It was widely used at the time of [24], but it was superseded by techniques based on implicit function methods, so it has sort of disappeared. Of course, 
one can also give a treatment of the convergence of our problem using implicit function theorems in Banach spaces too. Since the proof of convergence in our problem is only moderately more complicated than the case of the one-dimensional map in [24], we decided to include also a presentation of the majorant method.

Of course, at the time of [24], the method was used for numerical series and the goal was to estimate the absolute value. With a modern perspective, it is straightforward to remark that one can use it also for series of functions in a Banach space, and the role of the absolute value is taken by the norm.

The goal of the majorant method is to estimate a recursion of the form generated by (4.8), namely

$$
u_{\ell}=\Gamma_{\ell}\left(u_{1}, \ldots, u_{\ell-1}\right) \text {, }
$$

where $u_{\ell}$ are functions in a Banach space and $\Gamma_{\ell}$ is a polynomial. We expand $\Gamma_{\ell}$ as

$$
\Gamma_{\ell}\left(u_{1}, \ldots, u_{\ell-1}\right)=\sum_{k} a_{\ell k_{1} \ldots k_{\ell-1}} u_{1}^{k_{1}} \ldots u_{\ell-1}^{k_{\ell-1}}
$$

where $a_{\ell k_{1} \ldots k_{\ell-1}}$ are multilinear operators.

In our application, the polynomial $\Gamma_{\ell}$ is obtained from $(4.8)$ as $D_{b}^{-1}\left((\tilde{M})^{-1} \circ T_{\omega} S_{\ell}\right)$, where $D_{b}$ is given by $D_{b} u=b u-\lambda^{\ell} u \circ T_{\omega}$. We take $b=1$ in the first component, $b=\lambda$ in the second component and $S_{\ell}$ is the coefficient of order $\ell$ in the Taylor expansion (compare with (4.5)). The important point is that, for $\ell \geq 2, D_{b}^{-1}$ is a bounded operator and that the bound is independent of $\ell$.

The majorant method is based on the following elementary general result that we will later apply in our case for appropriate choices of the majorant recurrence.

Proposition 10. Assume that we can find a sequence of real polynomials $\Upsilon_{\ell}, \ell \geq 2$, with positive coefficients, say $\Upsilon_{\ell}\left(\sigma_{1}, \ldots, \sigma_{\ell-1}\right)=\sum_{k} b_{\ell k_{1} \ldots k_{\ell-1}} \sigma_{1}^{k_{1}} \ldots \sigma_{\ell-1}^{k_{\ell-1}}$, such that $\Upsilon_{\ell}$ is a majorant of $\Gamma_{\ell}$, namely

$$
\left|a_{\ell k_{1} \ldots k_{\ell-1}}\right| \leq b_{\ell k_{1} \ldots k_{\ell-1}} .
$$

Assume that $\sigma_{1} \geq\left\|u_{1}\right\|$. Denote by $\sigma_{\ell}$ the solution of the recursion:

$$
\sigma_{\ell}=\Upsilon_{\ell}\left(\sigma_{1}, \ldots, \sigma_{\ell-1}\right), \quad \ell \geq 2
$$

then, the solutions of the recursion (4.10) satisfy the inequality

$$
\left\|u_{\ell}\right\| \leq \sigma_{\ell}
$$

Proof. The proof of Proposition 10 is clearly by induction, using that $\Upsilon_{\ell}$ is monotone in all its arguments. By definition, (4.11) holds true for $\ell=1$. For $\ell \geq 2$, assume that 
$\left\|u_{j}\right\| \leq \sigma_{j}$ for $1 \leq j \leq \ell-1$; then, one has

$$
\begin{aligned}
\left\|u_{\ell}\right\| & =\left\|\Gamma_{\ell}\left(u_{1}, \ldots, u_{\ell-1}\right)\right\| \\
& \leq \sum_{k}\left|a_{\ell k_{1} \ldots k_{\ell-1}}\right| \sigma_{1}^{k_{1}} \ldots \sigma_{\ell-1}^{k_{\ell-1}} \\
& \leq \sum_{k} b_{\ell k_{1} \ldots k_{\ell-1}} \sigma_{1}^{k_{1}} \ldots \sigma_{\ell-1}^{k_{\ell-1}} \\
& =\Upsilon_{\ell}\left(\sigma_{1}, \ldots, \sigma_{\ell-1}\right) \equiv \sigma_{\ell} .
\end{aligned}
$$

To prove the convergence of the solution of (4.8) using Proposition 10 we make some choices for the majorant $\Upsilon_{\ell}$, so that we can show that $\left\|u_{\ell}\right\| \leq \sigma_{\ell}$, where $\sigma_{\ell} \leq \eta^{\ell}$ for some $\eta \in \mathbb{R}^{+}$. Equivalently (by Cauchy bounds), we show that $\sigma(z)=\sum_{j \geq 1} \sigma_{j} z^{j}$ is an analytic function in a neighborhood of $z=0$.

To this end, the first observation is that, because $f$ is analytic, then for some $\mu \in \mathbb{R}_{+}$, we have for $\alpha=\left(\alpha_{1}, \ldots, \alpha_{n}\right) \in \mathbb{Z}_{+}^{n}$ and for all $\theta \in \mathbb{T}_{\rho}$ :

$$
\frac{1}{\alpha !}\left|\left(D^{\alpha} f\right) \circ K(\theta)\right| \leq \mu^{|\alpha|}
$$

Since $S_{\ell}$ is the sum of terms composed by the above derivative of $f \circ K$ times some powers of $u_{j}$, applying the triangle inequality in the sum of terms defining the expression of $S_{\ell}$, due to (4.9) we see that we can take $\Upsilon_{\ell}$ to be the coefficient of order $\ell$ in $z$ in the expansion of

$$
\sum_{n \geq 2} \mu^{n}\left(\sum_{j \geq 1} \sigma_{j} z^{j}\right)^{n}=\frac{\left(\mu \sum_{j \geq 1} \sigma_{j} z^{j}\right)^{2}}{1-\mu \sum_{j \geq 1} \sigma_{j} z^{j}}
$$

(notice that the term for $n=1$ coincides with the linear term in $g_{n} \circ T_{\omega}$ appearing in $(4.5))$.

We write:

$$
\sigma(z)=\sigma_{1} z+\sigma^{>}(z)=\sigma_{1} z+\sum_{j \geq 2} \sigma_{j} z^{j}
$$

We see that the recursion $\sigma_{\ell}=\Upsilon_{\ell}\left(\sigma_{1}, \ldots, \sigma_{\ell-1}\right)$ is equivalent to the fact that $\sigma(z)$ satisfies

$$
\sigma^{>}(z)=\frac{(\mu \sigma(z))^{2}}{1-\mu \sigma(z)}
$$

or, equivalently,

$$
\sigma_{1} z+\sigma^{>}(z)=\frac{\left(\mu\left(\sigma_{1} z+\sigma^{>}(z)\right)\right)^{2}}{1-\mu \sigma_{1} z-\mu \sigma^{>}(z)}+\sigma_{1} z
$$


It is easy to see that, if we fix $\sigma_{1}$, one can find, by algebraic manipulations, a $\sigma^{>}(z)$ solving (4.13). This solution is clearly analytic in a neighborhood of $z=0$. By Proposition 10, we have that $\left\|u_{\ell}\right\| \leq \sigma_{\ell}$, so that the perturbative series converges in a small neighborhood.

\section{Extension of THE RESUlts TO QUASI-PERIODIC PERTURBATIONS}

The goal of this section is to present an elementary construction that allows to extend the results presented before to quasi-periodic perturbations. The motivation is that quasi-periodic perturbations of conformally symplectic systems have been widely considered in the literature (see $[5,13]$, where a one-dimensional dissipative system subject to a quasi-periodic forcing term has been considered).

The construction will be quite elementary and it is an analogue of the standard symplectic extensions considered very often in the treatment of periodic or quasi-periodic Hamiltonian systems.

5.0.1. Definitions. We recall that a quasi-periodic map $F$ and a quasi-periodic vector field $X$ are given, respectively, by:

$$
\begin{aligned}
F(x, \varphi) & =(f(x, \varphi), \varphi+\nu) \\
(\dot{x}, \dot{\varphi}) & =(X(x, \varphi), \nu),
\end{aligned}
$$

where $x$ lies in a phase space $\mathcal{P}, \varphi \in \mathbb{T}^{\ell}, \nu \in \mathbb{R}^{\ell}$ and $f: \mathcal{P} \times \mathbb{T}^{\ell} \rightarrow \mathcal{P}$. For flows, $X(x, \varphi) \in T_{x} \mathcal{P}, \varphi \in \mathbb{T}^{\ell}, \nu \in T_{\varphi} \mathbb{T}^{\ell}=\mathbb{R}^{\ell}$.

A quasi-periodic solution of (5.1) is an embedding $K$ of $\mathbb{T}^{d} \times \mathbb{T}^{\ell}$ into $\mathcal{P}$ satisfying

$$
\begin{aligned}
f(K(\theta, \varphi), \varphi) & =K(\theta+\omega, \varphi+\nu) \\
\omega \cdot \partial_{\theta} K(\theta, \varphi)+\nu \partial_{\varphi} K(\theta, \varphi) & =X(\theta, \varphi) .
\end{aligned}
$$

Notice that we are not assuming any irrationality condition on $\omega, \nu$, which could even be rationals. In particular, our quasi-periodic solutions include as special cases families of periodic solutions, for which the results remain valid. Other results (e.g., the KAM theory in $[7,8])$ require Diophantine conditions.

The quasi-periodic map $F$ is conformally symplectic, when $\mathcal{P}$ has a symplectic form $\Omega$ and, for every $\varphi$, defining $f_{\varphi}: \mathcal{P} \rightarrow \mathcal{P}$ by $f(x, \varphi)=f_{\varphi}(x)$, we have

$$
\left(f_{\varphi}\right)^{*} \Omega=\lambda \Omega \text {. }
$$

Similarly, a flow is conformally symplectic if for every $\varphi$ we have

$$
L_{X(\cdot, \varphi)} \Omega=\eta \Omega .
$$


Note that for a fixed $\varphi, X(\cdot, \varphi)$ is a vector field in $\mathcal{P}$ and $L$ is the Lie derivative in $\mathcal{P}$. Again, we remark that quasi-periodic flows are generated by quasi-periodic vector fields.

5.1. Main construction. The goal is now to show that all the above constructions embed naturally in a conformally symplectic system of higher dimension. In this way, we can apply the main result of Theorem 2 to the extended system and we can obtain results for our original system.

We consider $\tilde{\mathcal{P}}=\mathcal{P} \times \mathbb{T}^{n} \times \mathbb{R}^{n}$ and we equip it with the symplectic form $\tilde{\Omega}=\Omega \oplus$ $\sum_{j=1}^{n} d A_{j} \wedge d \varphi_{j}$. Given a quasi-periodic map $F$ as above, we extend it as $\tilde{\mathcal{P}}$ by

$$
\tilde{F}(x, \varphi, A)=(f(x, \varphi), \varphi+\nu, \lambda A) .
$$

Similarly, we extend a quasi-periodic flow by introducing $\tilde{X}$ as

$$
\tilde{X}(x, \varphi, A)=(X(x, \varphi), \nu, \eta A) .
$$

It is immediate to check that if $F$ (respectively, $X$ ) is quasi-periodic conformally symplectic, then $\tilde{F}$ (respectively, $\tilde{X}$ ) is conformally symplectic.

Given a quasi-periodic solution and $K$ as above, we extend it as (respectively, in the case of maps and in the case of flows)

$$
\tilde{K}(\theta, \varphi)=(K(\theta, \varphi), \varphi+\nu, \lambda A), \quad \tilde{K}(\theta, \varphi)=(K(\theta, \varphi), \nu, \eta A) .
$$

It is also immediate to realize that, if $K$ is a quasi-periodic solution for the map $F$, then

$$
\begin{aligned}
\tilde{F} \circ \tilde{K} & =\tilde{K} \circ T_{\omega, \nu} \\
\left(\omega \partial_{\theta}, \nu \partial_{\varphi}\right) K & =\tilde{X} \circ \tilde{K},
\end{aligned}
$$

where $T_{\omega, \nu}$ is such that $\tilde{K} \circ T_{\omega, \nu}(\theta, \varphi)=\tilde{K}(\theta+\omega, \varphi+\nu)$.

5.2. Some elementary consequences of the construction. It follows from the above construction that, if we are given a quasi-periodic solution of a quasi-periodic system, then we can determine a solution of the extended system.

Applying the main result, we can find a neighborbood of the orbit in which the system is conjugate to the linearized version, say $\tilde{F}_{0}$.

This leads to a non-autonomous linearization, when we eliminate the added variables in the extended system and, in particular, to the fact that the quasi-periodic solution is attractive.

Remark 11. Notice that we are not claiming that this construction suffices to extend the KAM theorem in [7] that shows stability of the quasi-periodic solutions. The fact is 
that the KAM theorem of [7] requires non-degeneracy assumptions that are not verified by the extended system and that are not created by the extension.

On the other hand, we mention that there is indeed a KAM theorem for quasi-periodic perturbations, but this requires more sophisticated arguments, than using the constructions presented here to reduce it to [7]. Some of such theorems have been presented in [14]. Another line of argument, close to that in [7] is to observe that perturbations of quasiperiodic systems are very special and one can develop for them a theorem with weaker non-degeneracy assumptions (compare with [8]).

5.2.1. Relations with the perturbative approach. In $[5,13]$ a one-dimensional dissipative system of the form

$$
\ddot{x}+\gamma \dot{x}+g(x)=f(\omega t)
$$

is studied, where $\gamma>0$ is the dissipative coefficient, $g: \mathbb{R} \rightarrow \mathbb{R}, f: \mathbb{T}^{n} \rightarrow \mathbb{R}$, are real analytic functions, $\omega \in \mathbb{T}^{n}$ is the forcing frequency. The analysis of this system is based on the study of the response solution, namely the solution which is quasi-periodic with frequency equal to the forcing frequency. For $\gamma$ large enough, the solution is explicitly constructed through a perturbative approach in $\varepsilon \equiv 1 / \gamma$, provided some conditions are fulfilled, precisely that $\omega$ satisfies the Bryuno condition and that there exists a zero $x_{0}$ of odd order $m$ of the equation $g(x)-\bar{f}=0$, being $\bar{f}$ the average of $f$. Under such assumptions, the response solution provides a local attractor (see [5, 13]).

In the geometrically natural language of Section 3.1, the response solution associated to a vector field $X^{t}$ is given by $D X^{t} \circ K(\theta)$. The automatic reducibility developed in [7]

shows that $D X^{t} \circ K(\theta)$ is conjugated to $\left(\begin{array}{cc}\mathrm{Id} & 0 \\ 0 & e^{\eta t} \mathrm{Id}\end{array}\right)$ and the conjugating matrix is the matrix $M$ given explicitly in (3.3). We note that the construction leading to (3.5) is nonperturbative, but it also makes sense when $K$ is substituted by a perturbative expansion (e.g., a formal sum of trees). We stress that the solution obtained in the present paper is very general and, in particular, it does not require any assumption on $\omega$ (like the Bryuno condition) or on the vector field.

\section{REFERENCES}

[1] R. Abraham and J. Robbin. Transversal mappings and flows. W. A. Benjamin, Inc., New YorkAmsterdam, 1967.

[2] L. F. A. Arbogast. Du Calcul des derivations. Levraut, Strasbourg, 1800. Available freely from Google Books.

[3] A. Banyaga. Some properties of locally conformal symplectic structures. Comment. Math. Helv., $77(2): 383-398,2002$. 
[4] A. Banyaga, R. de la Llave, and C. E. Wayne. Cohomology equations near hyperbolic points and geometric versions of Sternberg linearization theorem. J. Geom. Anal., 6(4):613-649 (1997), 1996.

[5] M. V. Bartuccelli, J. H. B. Deane, and G. Gentile. Globally and locally attractive solutions for quasi-periodically forced systems. J. Math. Anal. Appl., 328(1):699-714, 2007.

[6] R. P. Brent and H. T. Kung. Fast algorithms for manipulating formal power series. J. Assoc. Comput. Mach., 25(4):581-595, 1978.

[7] R. Calleja, A. Celletti, and R. de la Llave. KAM theory for conformally symplectic systems. 2011. MP_ARC \# 11-182.

[8] R. de la Llave and W. Alistair. KAM theory for perturbations with infinitely many frequencies. 2011. Preprint.

[9] R. de la Llave, J. M. Marco, and R. Moriyón. Canonical perturbation theory of Anosov systems and regularity results for the Livšic cohomology equation. Ann. of Math. (2), 123(3):537-611, 1986.

[10] F. Faa di Bruno. Note sur une nouvelle formule de calcul differentiel. Quart. Jour. of Pure and App. Math, 1:359-360, 1857.

[11] N. Fenichel. Asymptotic stability with rate conditions. Indiana Univ. Math. J., 23:1109-1137, 1973 $-1974$.

[12] N. Fenichel. Asymptotic stability with rate conditions. II. Indiana Univ. Math. J., 26(1):81-93, 1977.

[13] G. Gentile. Quasi-periodic motions in strongly dissipative forced systems. Ergodic Theory Dynam. Systems, 30(5):1457-1469, 2010.

[14] G. Gentile. Quasiperiodic motions in dynamical systems: review of a renormalization group approach. J. Math. Phys., 51(1):015207, 34, 2010.

[15] A. González-Enríquez, A. Haro, and R. de la Llave. Singularity theory of non-twist KAM tori. Memoirs of the AMS, 2012. MP_ARC \# 11-179, TO APPEAR.

[16] J. Guckenheimer. Isochrons and phaseless sets. J. Math. Biol., 1(3):259-273, 1974/75.

[17] A. Guillamon and G. Huguet. A computational and geometric approach to phase resetting curves and surfaces. SIAM J. Appl. Dyn. Syst., 8(3):1005-1042, 2009.

[18] A. Haro. Automatic differentiation tools in computational dynamical systems. Work in progress, 2011.

[19] M. Hirsch, J. Palis, C. Pugh, and M. Shub. Neighborhoods of hyperbolic sets. Invent. Math., 9:121$134,1969 / 1970$.

[20] G. Huguet and R. de la Llave. Computation of limit cycles and their isochrones: fast algorithms and their convergence. 2011. Preprint.

[21] G. Huguet, R. de la Llave, and Y. Sire. Fast numerical algorithms for the computation of invariant tori in hamiltonian systems. Disc. Cont. Dynam. Syst. A, 17(4):1309-1353.

[22] J. Moser. On the volume elements on a manifold. Trans. Amer. Math. Soc., 120:286-294, 1965.

[23] E. Nelson. Topics in Dynamics. I: Flows. Princeton University Press, Princeton, N.J., 1969.

[24] H. Poincaré. Note sur les propriétés de fonctions définies par les equations différentielles. Jour. de la Ec. Polyth., pages 13-26, 1878.

[25] C. L. Siegel and J. K. Moser. Lectures on Celestial Mechanics. Springer-Verlag, Berlin, 1995. Reprint of the 1971 translation.

[26] S. Sternberg. Local contractions and a theorem of Poincaré. Amer. J. Math., 79:809-824, 1957.

[27] A. Weinstein. Lectures on Symplectic Manifolds, volume 29 of CBMS Regional Conf. Ser. in Math. Amer. Math. Soc., Providence, 1977.

[28] A. T. Winfree. Patterns of phase compromise in biological cycles. J. Math. Biol., 1(1):73-95, $1974 / 75$. 